\title{
Aquaporin 4 expression is downregulated in large bovine ovarian follicles
}

\author{
Chang-Woon Kim ${ }^{1,2}$, Eun-Ju Choi ${ }^{1}$, Eun-Jin Kim ${ }^{1}$, Adrian S. Siregar ${ }^{1,3}$, Jaehee Han ${ }^{1}$ and Dawon Kang ${ }^{1,3, *}$ \\ ${ }^{1}$ Department of Physiology and Institute of Health Sciences, College of Medicine, Gyeongsang National University, Jinju \\ 52727, Korea \\ ${ }^{2}$ Department of Obstetrics and Gynecology, Samsung Changwon Hospital, Sungkyunkwan University School of \\ Medicine, Changwon 51353, Korea \\ ${ }^{3}$ Department of Convergence Medical Science, Gyeongsang National University, Jinju 52727, Korea
}

Received October 23, 2020

Revised December 1, 2020

Accepted December 7, 2020

\section{*Correspondence}

Dawon Kang

E-mail: dawon@gnu.ac.k

ORCID

https://orcid.org/0000-0001-7402-7298

\begin{abstract}
Aquaporin channels (AQPs) are known to play an important role in the development of ovarian follicles through their function in water transport pathways. Compared to other AQPS, research on the role of AQP4 in female reproductive physiology, particularly in cattle, remains limited. In our previous study, gene chip microarray data showed a downregulation of AQP4 in bovine cystic follicles. This study was performed to validate the AQP4 expression level at the protein level in bovine follicles using immunohistochemistry, Western blotting, and immunoprecipitation assays. Immunostaining data showed that AQP4 was expressed in granulosa and theca cells of bovine ovarian follicles. The ovarian follicles were classified according to size as small $(<10 \mathrm{~mm}$ ) or large $(>25 \mathrm{~mm})$ in diameter. Consistent with earlier microarray data, semi-quantitative PCR data showed a decrease in AQP4 mRNA expression in large follicles. Western blot analysis showed a downregulation of the AQP4 protein in large follicles. In addition, AQP4 was immunoprecipitated and blotted with anti-AQP4 antibody in small and large follicles. Accordingly, AQP4 exhibited a low expression in large follicles. These results show that AQP4 is downregulated in bovine ovarian large follicles, suggesting that the downregulation of AQP4 expression may interfere with follicular water transport, leading to bovine follicular cysts.
\end{abstract}

Keywords: aquaporin 4, cattle, granulosa cells, ovarian follicle, theca cells

\section{INTRODUCTION}

The flow of water and ions across the cell membrane serves as an important regulator in a variety of cellular processes, including ovarian folliculogenesis, which is characterized by the formation and expansion of the fluid-filled antrum (McConnell et al., 2002). Aquaporin channels (AQPs) transport water molecules into and out of cells, including water-permeable classic AQP (AQP0-2, $4-6,8)$, aquaglyceroporins (AQP3, 7, 9, 10) permeable to water and small non-electrolytic molecules (glycerol, urea, purines, and pyrimidines), and superaquaporins (AQP11, 12) with uncertain permeability (Zhu et al., 2015). It has been suggested that AQP isoforms (AQPs) play an important role in ovarian follicle development, because they function as a water transport pathway in ovarian follicles (McConnell et al., 2002; Su et al., 2013; Skowronska et al., 2015). Of the 13 AQPs (AQP 0-12), 11 isoforms, except $\mathrm{AQP0}$ and 10, are found in the female mammalian reproductive systems of humans, rodents, sheep, pigs, horses, 
and dogs (Zhu et al., 2015). Compared to other AQPs, there is still limited research available on the role of AQP4 in female reproductive physiology, particularly in humans and cattle.

AQP4 belongs to the water-permeable classic AQP family and is the most abundantly expressed channel in the central nervous system (Mader and Brimberg, 2019) and astrocytes closest to the vessel (Papadopoulos et al., 2004). Based on its expression pattern, AQP4 is involved in controlling brain water homeostasis and bidirectional fluid exchange because of its particularly high expression at the blood brain barrier (BBB) and blood cerebrospinal fluid (CSF) barrier (Mader and Brimberg, 2019). AQP4-deficient mice show an increase in brain edema (Papadopoulos et al., 2004). In addition, AQP4 deficiency induces sex-specific differences in the transmission of neurotransmitters in the brain, and decreases the serum estradiol $\left(\mathrm{E}_{2}\right)$ and progesterone $\left(\mathrm{P}_{4}\right)$ levels in mice (Fan et al., 2005; Sun et al., 2007; Zeng et al., 2007). These reports suggested that AQP4 may be related to reproductive function because AQP4 regulates the functions of sex hormones in the brain.

In the female reproductive system, numerous studies have demonstrated the role of AQP4 in rodent models. In terms of reproductive physiology, humans, cattle, and mice share characteristics, but there are differences in gene expression patterns, biophysical parameters, intrinsic factors in the regulation of embryonic development, and embryo metabolism (Ménézo and Hérubel, 2002; Mateo-Otero et al., 2020). Compared to mice, there are many similar properties between humans and cattle; biochemical and intrinsic paternal and maternal control mechanisms in preimplantation embryos, microregulation in the final stage of oocyte maturation, and interactions between the embryo and corpus luteum (Ménézo and Hérubel, 2002). Expression of AQP4 has been reported in human and chicken ovarian granulosa and theca cells (Thoroddsen et al., 2011; Nowak et al., 2017). Our previous study reported that AQP4 mRNA was downregulated in bovine ovarian large follicles (larger than $25 \mathrm{~mm}$ in diameter, > $25 \mathrm{~mm}$ ) (Tak et al., 2010). Although AQP4 plays an important role in ovarian folliculogenesis, AQP4's role has not been studied in bovine ovaries since our previous report. The study of AQPs' roles in the bovine ovary is valuable because it can be helpful to address different bovine reproductive issues and to provide basic data for un- derstanding its possible role in human ovaries. This study was performed to analyze the expression level of AQP4 at the protein level in bovine follicles.

\section{MATERIALS AND METHODS}

\section{Ethics}

The Korean legislature makes an exception to the ethical approval of experiments with bovine ovaries obtained in slaughterhouses. All experiments were conducted according to the guidelines of the Institutional Animal Care and Use Committee of Gyeongsang National University. The experimental materials and methods were used similarly to previous studies conducted in this laboratory (Siregar et al., 2019; Choe et al., 2010).

\section{Isolation of granulosa cells}

The ovaries of Korean cattle (Hanwoo) were collected from a slaughterhouse and transported to the laboratory in phosphate buffered saline (PBS) at $35-39^{\circ} \mathrm{C}$. Follicles were isolated from the ovaries at the follicular phase, where there were no corpora lutea in either the right or left ovaries. The ovarian follicles were classified into sizes as small $(<10 \mathrm{~mm}, 5$ to $10 \mathrm{~mm})$ and large $(>25 \mathrm{~mm}$ in diameter). The isolation procedures were performed as previously described (Choe et al., 2010). Briefly, follicular fluid (FF) was carefully aspirated from small and large follicles with a $10 \mathrm{~mL}$ syringe fitted with an 18 or 23 gauge needle; the fluid was centrifuged at $1,750 \times \mathrm{g}$ for $10 \mathrm{~min}$, and the resultant pellets were used for identification of AQP4 expression. The resultant pellets contained the most granulosa cells. In FF isolated from large follicles, the concentration of $E_{2}$ was high, and the concentration of testosterone was low.

\section{Hematoxylin and eosin (H\&E) staining}

The H\&E staining procedures were performed as previously described (Choe et al., 2010). Briefly, ovaries were washed with PBS, fixed with $4 \%(\mathrm{w} / \mathrm{v})$ paraformaldehyde in $0.1 \mathrm{M}$ PBS, processed into paraffin-embedded tissue blocks, and cut to $4 \mu \mathrm{m}$ thickness. The ovarian sections were air-dried on gelatin-coated slides, and deparaffinized. The deparaffinized tissue sections were washed with tap water for $5 \mathrm{~min}$, immersed in hematoxylin for 10 min, and confirmed complete staining of hematoxylin in tap water, followed by eosin staining for $3 \mathrm{~min}$. The tissue 
sections were dehydrated through a graded series of alcohols ( $70 \%$ to $100 \%$ ethanol, 3 min each), cleared in xylene, and mounted with a mounting medium (Permount ${ }^{\circledR}$, Fisher Scientific, Geel, Belgium). We analyzed and photographed stained sections with a $\mathrm{BH}-2$ microscope (Olympus, Tokyo, Japan) and a high-resolution video camera (DXC-960 MD; Sony, Tokyo, Japan). Five sections from each sample were evaluated.

\section{Immunohistochemistry (IHC)}

The ovarian sections were used for immunostaining to identify the expression and localization of AQP4. The immunostaining procedures were performed as previously described (Siregar et al., 2019). Briefly, deparaffinized tissue sections were permeabilized with $0.2 \%$ Triton X-100 for $10 \mathrm{~min}$ at room temperature. After three washes in PBS, the sections were incubated with blocking buffer (10\% normal goat serum in $0.1 \mathrm{M} \mathrm{PBS}$ ) for $60 \mathrm{~min}$ at room temperature. Without washing, the sections were then incubated with rabbit polyclonal anti-AQP4 (1:200 dilutions, Abcam, Cambridge, UK) primary antibody overnight at $4^{\circ} \mathrm{C}$. After three washes in PBS, the sections were incubated in the dark for $1.5 \mathrm{~h}$ with FITC-conjugated anti-rabbit IgG secondary antibody (Abcam) diluted at 1:400 in PBS. Finally, the sections were washed three times in PBS and stained with $4^{\prime}, 6$-diamidino-2-phenylindole (DAPI) for nuclei staining. The stained sections were wet-mounted with Gel/Mount ${ }^{\mathrm{TM}}$ (Biomeda Corp., Foster City, CA, USA) and observed using a confocal laser-scanning microscope (Olympus, Tokyo, Japan).

\section{Semiquantitative reverse transcriptase (RT) - polymerase chain reaction (PCR)}

Total RNA was extracted from the granulosa cells with TRIzol reagent (Thermo Fisher Scientific, Eugene, OR, USA) according to the manufacturer's instructions. First strand cDNAs were synthesized from total RNA using oligo dT (SuperScript ${ }^{\mathrm{TM}}$ First-Strand Synthesis System for RT-PCR, Invitrogen, Carlsbad, CA, USA) according to the manufacturer's instructions. The first-strand cDNA synthesized from the total RNA $(3 \mu \mathrm{g})$ was quantified using a spectrophotometer (NanoDrop ${ }^{\circledR}$ ND-1000, NanoDrop Technologies, Wilmington, DE, USA). The quantified cDNA was used as a template. The RT-PCR procedures were performed as previously described (Siregar et al., 2019). The PCR steps included initial denaturation at $94^{\circ} \mathrm{C}$ for $5 \mathrm{~min}$, then 32 cycles at $94^{\circ} \mathrm{C}$ for $20 \mathrm{sec}, 55^{\circ} \mathrm{C}$ for 20 sec, $72^{\circ} \mathrm{C}$ for $20 \mathrm{sec}$, and a final extension step at $72^{\circ} \mathrm{C}$ for $10 \mathrm{~min}$. The PCR products amplified with specific primers for AQP4 (NM_181003, sense 5'-TTCCCTAAAATACATGCAAGA-3', antisense 5'-TAATTCATGGAAATCAACCAC-3', expected size $490 \mathrm{bp}$ ) were separated in $1.5 \%$ agarose gel stained with ethidium bromide. The PCR bands were extracted and directly sequenced with an ABI PRISM ${ }^{\circledR}$ 3100-Avant Genetic Analyzer (Applied Biosystems, Foster City, CA, USA).

\section{Western blot analysis}

Western blot analysis was performed as previously described (Choe et al., 2010). Briefly, granulosa cells were homogenized in a lysis buffer (RIPA buffer, Cell Signaling Technology, Danvers, MA, USA) by sonication twice with a pulse of $20 \mathrm{sec}$ on ice. The cell lysates were incubated for $30 \mathrm{~min}$ on ice with intermittent vortexing and were clarified by centrifugation at 13,000 rpm $(16,609 \times \mathrm{g}$, Micro $17 \mathrm{TR}$, Hanil, Korea) for $30 \mathrm{~min}$ at $4^{\circ} \mathrm{C}$. After centrifugation, the supernatant was separated and stored at $-70^{\circ} \mathrm{C}$ until use. Protein concentration was quantified using a Pierce bicinchoninic acid (BCA) Protein Assay Kit (Thermo Fisher Scientific, Waltham, MA, USA). Equal volumes of the supernatant and $2 \times$ sodium dodecyl sulfate (SDS) sample buffer were mixed, loaded on $10 \%$ SDS-polyacrylamide gel, and separated by electrophoresis for $120 \mathrm{~min}$ at $120 \mathrm{~V}$. Then, the gel was transferred to a polyvinylidene difluoride membrane (Millipore, Billerica, MA, USA) for 1 $\mathrm{h}$ at $100 \mathrm{~V}$ using a wet transfer system (Bio-Rad, Hercules, CA, USA). The blots were blocked for $1 \mathrm{~h}$ with $5 \%$ fatfree milk and $0.05 \%$ Tween 20 in TBS and immunoblotted with anti-AQP4 monoclonal (Abcam, Cambridge, UK) or polyclonal (Santa Cruz Biotechnology, Inc., CA, USA) antibody, at 1:1000 dilutions at $4^{\circ} \mathrm{C}$ overnight. Subsequently, horseradish peroxidase (HRP)-conjugated goat anti-rabbit or goat anti-mouse secondary antibody (1:3000; assay designs, Ann Arbor, MI, USA) was immunoreacted at room temperature for $1 \mathrm{~h}$. Finally, the antigens were detected by enhanced chemiluminescence (ECL Plus kit; ELPIS, Taejeon, Korea) according to the manufacturer's instructions.

\section{Immunoprecipitation with Western blot analysis}

Granulosa cells were homogenized in RIPA buffer. The homogenate was incubated for $1 \mathrm{~h}$ at $4^{\circ} \mathrm{C}$ with intermit- 
tent vortexing and centrifuged at $16,609 \times \mathrm{g}$ for $30 \mathrm{~min}$. The supernatant was pre-cleared with Protein A/G PLUSagarose (Santa Cruz Biotechnology) overnight at $4^{\circ} \mathrm{C}$. The pre-cleared supernatant was incubated with the $1 \mu \mathrm{g}$ of AQP4 antibody for $1 \mathrm{~h}$ at $4^{\circ} \mathrm{C}$ and then was incubated with Protein A/G PLUS-agarose overnight at $4^{\circ} \mathrm{C}$. The agarose beads were washed three times with cold RIPA buffer, and the proteins were eluted by boiling in SDS-polyacrylamide gel electrophoresis (SDS-PAGE) sampling buffer. Proteins were separated by SDS-PAGE, and the gel was transferred to a PVDF membrane. For Western blot analysis, the above protocol was followed with an anti-AQP4 monoclonal antibody (1:1,000 dilutions, Abcam) and an anti-actin polyclonal antibody (1:1,000 dilutions, Sigma).

\section{Data analysis and statistics}

The luminescent image analyzer LAS-4000 (Fujifilm Corp., Tokyo, Japan) captures images of agarose gel and blot. The bands obtained from RT-PCR and Western blot were quantified by Sigma Gel image analysis software (version 1.0, Jandel Scientific, CA, USA) or Quantity One software (version 4.6.3) attached to a GS-800 Calibrated densitometer (Bio-Rad, Hercules, CA, USA). Relative mRNA and protein levels were calculated by comparing them to the amount of glyceraldehyde-3-phosphate dehydrogenase $(G A P D H)$ and $\beta$-actin, respectively. The data are represented as the mean \pm S.D. Significant differences between groups were evaluated using paired Student's $t$-tests (OriginPro 8, Northampton, MA, USA). A value of $p<0.05$ was considered to be significant.

\section{RESULTS}

\section{Expression of AQP4 in bovine ovary}

Immunohistochemistry was performed to identify AQP4 expression in bovine ovaries, which do not contain follicles larger than $25 \mathrm{~mm}$ (> $25 \mathrm{~mm}$ ), and the corpus luteum. H\&E staining exhibited granulosa and theca cell layers in the ovarian section (Fig. 1A). Immunostaining data showed that AQP4 was expressed in granulosa and theca cells (Fig. 1B). AQP4 was also detected in ovarian stroma. The AQP4 expression was visualized in green on immunostaining.

\section{Downregulation of AQP4 expression level in granulosa cells isolated from large follicles}

Ovarian follicles were classified according to size as smaller than $10 \mathrm{~mm}(<10 \mathrm{~mm})$ or larger than $25 \mathrm{~mm}(>25$ $\mathrm{mm})$ in diameter. Semi-quantitative PCR data showed a decrease in AQP4 mRNA expression in follicles $>25 \mathrm{~mm}$ (Fig. 2A). The AQP4 mRNA expression was significantly decreased by $48.4 \pm 12.7 \%$ in follicles $>25 \mathrm{~mm}(p<0.05$, Fig. 2B).

Western blot analysis showed a downregulation of AQP4 protein expression in follicles $>25 \mathrm{~mm}$. The AQP4 expression levels were significantly decreased in follicles $>$ $25 \mathrm{~mm}$ by $85.3 \pm 9.4 \%$ ( $p<0.05$, Fig. $3 \mathrm{~A})$. To validate the low expression of AQP4 in follicles $>25 \mathrm{~mm}, \mathrm{AQP} 4$ was immunoprecipitated and blotted with anti-AQP4 antibody in follicles $<10 \mathrm{~mm}$ and $>25 \mathrm{~mm}$. Accordingly, AQP4 exhibited low expression in follicles $>25 \mathrm{~mm}$ (Fig. 3B).
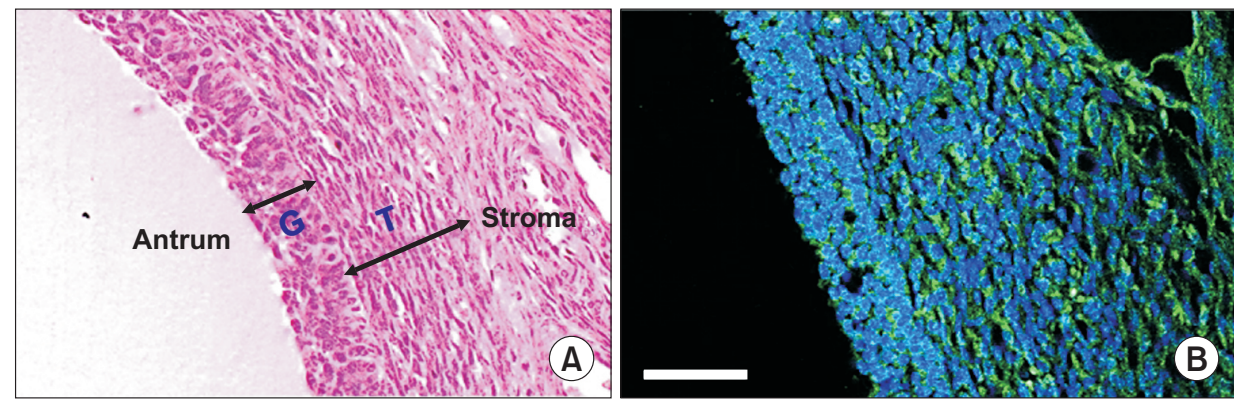

Fig. 1. Confirmation of the expression of AQP4 protein in bovine ovarian section by immunostaining. (A) H\&E staining of bovine ovarian section. The ovary with follicles $<10 \mathrm{~mm}$ in diameter shows antrum, granulosa cell layers (G), theca cell layers (T), and stroma. (B) Immunohistochemical staining of AQP4. AQP4 was visualized using an AQP4 antibody, followed by a secondary antibody conjugated with fluorescein isothiocyanate (FITC, blue). The nucleus was stained by DAPI (blue). Scale bar, $50 \mu \mathrm{m}$ 

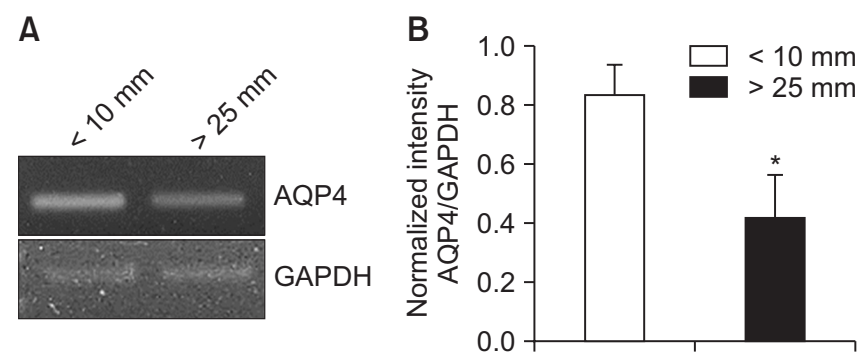

Fig. 2. AQP4 mRNA expression downregulated in large ovarian follicles (> $25 \mathrm{~mm}$ ). (A) Semi-quantitative PCR data for AQP-4 in follicles smaller than $10 \mathrm{~mm}(<10 \mathrm{~mm})$ and $>25 \mathrm{~mm}$. (B) Normalized AQP-4 mRNA expression levels in follicles $<10 \mathrm{~mm}$ and $>25 \mathrm{~mm}$ in size. The AQP4 mRNA expression level was normalized to that of GAPDH. Each bar represents the mean \pm SD of four independent experiments. ${ }^{*} p<0.05$ compared to follicles $<10 \mathrm{~mm}$.

A

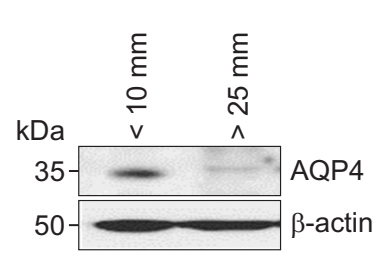

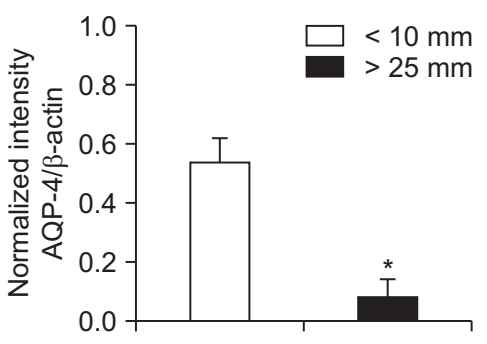

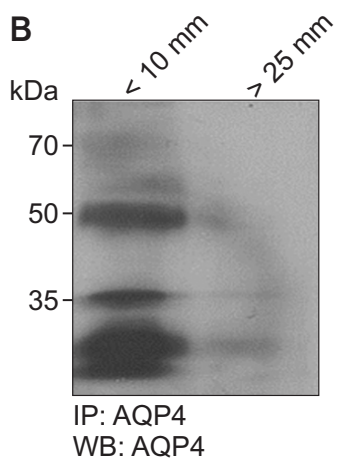

Fig. 3. AQP4 protein expression downregulated in large ovarian follicles (> $25 \mathrm{~mm}$ ). (A) Western blot analysis for AQP4. Relative AQP4 protein level was determined by comparing it to $\beta$-actin level. Each bar represents the mean \pm SD of four independent experiments. ${ }^{*} p<0.05$ compared to follicles $<10 \mathrm{~mm}$. (B) Immunoprecipitation assay for AQP4. Three independent experiments were performed ( $\mathrm{n}=3$ ). Follicular cell lysates were subjected to immunoprecipitation with an anti-AQP4 antibody. The lysates were resolved by SDS-PAGE, transferred to a PVDF membrane, and immunoblotted with Anti-AQP4 antibody. Equal amounts $(30 \mu \mathrm{g})$ of total protein were loaded on each well of the gels.

\section{DISCUSSION}

Ovarian follicles express AQP4 in granulosa and theca cells. Compared to theca cells in humans, cells in which AQP4 is mainly expressed differ depending on the species. AQP4 expression level is higher in granulosa cells (Thoroddsen et al., 2011), but compared to theca cells in chicken, its expression level is lower in granulosa cells (Nowak et al., 2017). Our study mainly confirmed the expression of AQP4 in granulosa cells without examining the difference in AQP4 expression levels between granulosa and theca cells. AQP4 expression level is changed during follicle development regardless of species. AQP4 expression gradually decreases from pre-ovulatory to the early ovulatory phase in human (Thoroddsen et al., 2011). Nowak et al. (2017) report that the expression of AQP4 mRNA progressively decreased along with the development of chicken ovarian follicles (Nowak et al., 2017). Our study also showed that AQP4 mRNA and protein ex- pression decreased in large follicles, which exhibited an expansion of fluid-filled antrum.

AQPs expression level is affected by ovarian hormones, such as $E_{2}$ or $P_{4}$ (Richard et al., 2003). AQP4 expression is upregulated by testosterone in cultured astrocytes ( $\mathrm{Gu}$ et al., 2003), but it does not respond to $E_{2}$ or $P_{4}$ in the mouse uterus (Richard et al., 2003). Our previous study demonstrated an increase in $\mathrm{E}_{2}$ concentration and a decrease in testosterone concentration in follicular fluid obtained from large follicles (> $25 \mathrm{~mm}$ in diameter) (Choe et al., 2010). Testosterone concentration reduced in ovarian large follicles may negatively affect the level of AQP4 expression.

In the normal ovulatory cycle, pre-ovulatory mature follicle dimensions are between 17 and $25 \mathrm{~mm}$ in diameter (Hackelöer et al., 1979). A maximum follicle size > $25 \mathrm{~mm}$ is considered large, which is estimated to be a biomarker of ovarian pathology, such as infertility and possible miscarriage (Achebe, 2019). In cattle, the structure of an ova- 
ry with a cavity larger than $25 \mathrm{~mm}$ in diameter represents a cystic ovary, which causes reproductive failure (Kengaku et al., 2007). In addition, follicular cystic ovaries show high $\mathrm{E}_{2}$ concentration and low testosterone concentration, while luteal cystic ovaries show low $\mathrm{E}_{2}$ concentration and high $\mathrm{P}_{4}$ concentration (Choe et al., 2008). The ovaries with large follicles used in this study represent follicular cystic ovaries, with high concentration of $E_{2}$ and low concentration of testosterone. In large ovarian follicles, low AQP4 expression may be linked to follicular cysts in cattle. AQP4 is suggested to function as a water transport pathway in ovarian follicles, and therefore plays a critical role in ovarian follicular development (McConnell et al., 2002; Su et al., 2013; Skowronska et al., 2015). AQP4deficient mice show subfertility with defective folliculogenesis, reduced corpus luteum formation, and decreased uterine response to gonadotropins (Sun et al., 2009).

It is suggested that most AQPs expressed in granulosa and theca cells are important in the formation of antrum during follicular development. The prominent feature of the antrum is the formation of follicular fluid. Water transport into the antral cavity of the follicle could be greater than movement into granulosa and theca cells. Low expression of AQP4 could be likely linked to decrease in water transport into granulosa and theca cells. Relatively, other AQP channels could contribute to water transport into the antral cavity of the follicle. Most single $\mathrm{AQP}$ isoform-deficient mice have phenotypes that do not differ substantially from the wild-type (Verkman, 2009) by compensatory mechanisms from other AQPs. Therefore, imbalanced water transport according to the low expression level of AQP4 may induce formation of excessively enlarged antrum. Abnormal ovarian and uterine AQP expression including AQP4 may be associated with infertility. In line with our findings, the expression levels of AQPs were decreased in ovaries showing reproductive failure. AQP9 expression levels have been shown to be lower in the granulosa cells of women with polycystic ovary syndrome (PCOS) than in controls (Qu et al., 2010). AQP8-deficient mice show multi-oocyte follicles (MOFs) containing two or more oocytes within the same follicle (Su et al., 2013), which do not contain multiple healthy oocytes (Christensen et al., 2015).

There are some limitations in this study. We could not clearly analyze the difference in AQP4 expression levels between granulosa and theca cells. In our previous study, we demonstrated high concentrations of $E_{2}$ and low concentrations of testosterone in large follicles (Choe et al., 2010). However, we did not address the direct effects of ovarian hormones on AQP4 expression in this study. Further study is needed to address the relationships among AQP4 expression levels, ovarian hormones, and follicular cysts.

\section{CONCLUSION}

In conclusion, AQP4 mRNA and protein were downregulated in the large ovarian follicles (> $25 \mathrm{~mm}$ in diameter) of Korean cattle. Our results suggest that downregulation of AQP4 expression could lead to bovine follicular cysts by interfering with follicular water transport.

\section{CONFLICTS OF INTEREST}

No potential conflict of interest relevant to this article was reported.

\section{ACKNOWLEDGEMENTS}

This work was supported by the "On-Site Cooperative Agriculture Research Project (20080101-080-057001-01-00, RDA)", by the National Research Foundation of Korea (MSIT, NRF-2015R1A-5A-2008833; NRF2018R1A2B6001446), and by the grant from Institute of Health Sciences of Gyeongsang National University (IHC GNU-2018-01). We thank Dr. Changyong Choe for providing the bovine ovary sample and Eun-Sook Kim for technical assistance. This work was partially from ChangWoon Kim's thesis for the degree of Doctor of Philosophy (Alteration of the Gene Expression Patterns in follicular cyst of bovine ovary, 2016, Department of Medicine, Gyeongsang National University).

\section{AUTHOR CONTRIBUTIONS}

Conceptualization: Chang-Woon Kim, Dawon Kang

Data curation: Eun-Ju Choi, Eun-Jin Kim, Adrian S. Siregar

Formal analysis: Eun-Ju Choi, Eun-Jin Kim, Adrian S. Siregar, Dawon Kang

Funding acquisition: Dawon Kang/Investigation: Eun-Ju Choi, Adrian S. Siregar

Methodology: Eun-Ju Choi, Adrian S. Siregar, Dawon Kang Project administration: Eun-Jin Kim/Resources: Chang- 
Yong Choe (acknowledgement)

Software: Eun-Ju Choi, Eun-Jin Kim, Adrian S. Siregar, Dawon Kang

Supervision: Jaehee Han, Dawon Kang/Validation: EunJin Kim, Dawon Kang

Visualization: Eun-Ju Choi, Adrian S. Siregar, Dawon Kang

Writing - original draft: Dawon Kang/Writing - review \& editing: Dawon Kang

\section{AUTHOR'S POSITION AND ORCID NO.}

\author{
CW Kim, Professor, \\ https://orcid.org/0000-0002-6663-0764 \\ EJ Choi, PhD Student, \\ https://orcid.org/0000-0002-9086-8801 \\ EJ Kim, Postdoctoral Researcher, \\ https://orcid.org/0000-0003-1068-9334 \\ AS Siregar, PhD Student, \\ https://orcid.org/0000-0001-7069-4202 \\ J Han, Professor, \\ https://orcid.org/0000-0002-3523-008X \\ D Kang, Professor, \\ https://orcid.org/0000-0001-7402-7298
}

\section{REFERENCES}

Achebe FU. 2019. Relationship of maximum follicular size, age of woman, and reproductive implications in women attending fertility clinic in St. Margaret's Hospital, Lokoja, using Creighton Model FertilityCare ${ }^{\mathrm{TM}}$ System and NaProTECHNOLOGY. Niger. J. Gen. Pract. 17:51-63.

Choe C, Cho YW, Kim CW, Son DS, Han J, Kang D. 2010. Identification of differentially expressed genes in bovine follicular cystic ovaries. Korean J. Physiol. Pharmacol. 14:265-272.

Choe C, Son DS, Cho SR, Kim HJ, Choi SH, Kang D. 2008. Alteration in concentrations of blood urea nitrogen and sex steroid hormone in Korean cattle with reproductive disorders. J. Emb. Trans. 23:59-64.

Christensen AP, Patel SH, Grasa P, Christian HC, Williams SA. 2015. Oocyte glycoproteins regulate the form and function of the follicle basal lamina and theca cells. Dev. Biol. 401:287298.

Fan Y, Zhang J, Sun XL, Gao L, Zeng XN, Ding JH, Cao C, Niu L, Hu G. 2005. Sex- and region-specific alterations of basal amino acid and monoamine metabolism in the brain of aquaporin-4 knockout mice. J. Neurosci. Res. 82:458-464.

Gu F, Hata R, Toku K, Yang L, Ma YJ, Maeda N, Sakanaka M, Tanaka J. 2003. Testosterone up-regulates aquaporin-4 expression in cultured astrocytes. J. Neurosci. Res. 72:709-715.

Hackelöer BJ, Fleming R, Robinson HP, Adam AH, Coutts JR.
1979. Correlation of ultrasonic and endocrinologic assessment of human follicular development. Am. J. Obstet. Gynecol. 135:122-128.

Kengaku K, Tanaka T, Kamomae H. 2007. Changes in the peripheral concentrations of inhibin, follicle-stimulating hormone, luteinizing hormone, progesterone and estradiol17beta during turnover of cystic follicles in dairy cows with spontaneous follicular cysts. J. Reprod. Dev. 53:987-993.

Mader S and Brimberg L. 2019. Aquaporin-4 water channel in the brain and its implication for health and disease. Cells 8:90.

Mateo-Otero Y, Sánchez JM, Recuero S, Bagés-Arnal S, McDonald M, Kenny DA, Yeste M, Lonergan P, Fernandez-Fuertes B. 2020. Effect of exposure to seminal plasma through natural mating in cattle on conceptus length and gene expression. Front. Cell Dev. Biol. 8:341.

McConnell NA, Yunus RS, Gross SA, Bost KL, Clemens MG, Hughes FM Jr. 2002. Water permeability of an ovarian antral follicle is predominantly transcellular and mediated by aquaporins. Endocrinology 143:2905-2912.

Ménézo YJ and Hérubel F. 2002. Mouse and bovine models for human IVF. Reprod. Biomed. Online 4:170-175.

Nowak M, Grzesiak M, Saito N, Kwaśniewska M, Sechman A, Hrabia A. 2017. Expression of aquaporin 4 in the chicken ovary in relation to follicle development. Reprod. Domest. Anim. 52:857-864.

Papadopoulos MC, Manley GT, Krishna S, Verkman AS. 2004. Aquaporin-4 facilitates reabsorption of excess fluid in vasogenic brain edema. FASEB J. 18:1291-1293.

Qu F, Wang FF, Lu XE, Dong MY, Sheng JZ, Lv PP, Ding GL, Shi BW, Zhang D, Huang HF. 2010. Altered aquaporin expression in women with polycystic ovary syndrome: hyperandrogenism in follicular fluid inhibits aquaporin-9 in granulosa cells through the phosphatidylinositol 3-kinase pathway. Hum. Reprod. 25:1441-1450.

Richard C, Gao J, Brown N, Reese J. 2003. Aquaporin water channel genes are differentially expressed and regulated by ovarian steroids during the periimplantation period in the mouse. Endocrinology 144:1533-1541.

Siregar AS, Nyiramana MM, Kim EJ, Shin EJ, Kim CW, Lee DK, Hong SG, Han J, Kang D. 2019. TRPV1 is associated with testicular apoptosis in mice. J. Anim. Reprod. Biotechnol. 34:311-317.

Skowronska A, Mlotkowska P, Eliszewski M, Nielsen S, Skowronski MT. 2015. Expression of aquaporin 1, 5 and 9 in the ovarian follicles of cycling and early pregnant pigs. Physiol. Res. 64:237-245.

Su W, Guan X, Zhang D, Sun M, Yang L, Yi F, Hao F, Feng X, Ma T. 2013. Occurrence of multi-oocyte follicles in aquaporin 8-deficient mice. Reprod. Biol. Endocrinol. 11:88.

Sun XL, Ding JH, Fan Y, Zhang J, Gao L, Hu G. 2007. Aquaporin 4 regulates the effects of ovarian hormones on monoamine neurotransmission. Biochem. Biophys. Res. Commun. 353:457-462.

Sun XL, Zhang J, Fan Y, Ding JH, Sha JH, Hu G. 2009. Aquaporin-4 deficiency induces subfertility in female mice. Fertil. 
Steril. 92:1736-1743.

Tak HM, Han J, Kang D. 2010. Fibrinogen mRNA expression upregulated in follicular cyst of Korean cattle. J. Emb. Trans. 25:29-34.

Thoroddsen A, Dahm-Kähler P, Lind AK, Weijdegård B, Lindenthal B, Müller J, Brännström M. 2011. The water permeability channels aquaporins 1-4 are differentially expressed in granulosa and theca cells of the preovulatory follicle during precise stages of human ovulation. J. Clin. Endocrinol.
Metab. 96:1021-1028.

Verkman AS. 2009. Knock-out models reveal new aquaporin functions. Handb. Exp. Pharmacol. (190):359-381.

Zeng XN, Sun XL, Gao L, Fan Y, Ding JH, Hu G. 2007. Aquaporin-4 deficiency down-regulates glutamate uptake and GLT1 expression in astrocytes. Mol. Cell. Neurosci. 34:34-39.

Zhu C, Jiang Z, Bazer FW, Johnson GA, Burghardt RC, Wu G. 2015. Aquaporins in the female reproductive system of mammals. Front. Biosci. (Landmark Ed.) 20:838-871. 